\title{
EDITORIAL Turning fluorescence into black and white
}

\author{
Peter E. Fecci, MD, PhD, Ranjith Babu, MD, D. Cory Adamson, MD, PhD, \\ and John H. Sampson, MD, PhD, MBA, MHSc
}

Division of Neurosurgery, Duke University Medical Center, Durham, North Carolina

$\mathrm{W}$

HILE questions persist as to the delineable influence for extent of glioma resection (EOR) on outcome measures such as quality of life and survival, equally persistent are our efforts to improve rates of resection, despite the controversy. Ultimately, there are 2 pervasive and recognizable barriers to the "gross-total resection" (GTR): insecurity regarding the borders between tumor and normal tissue, and fear of the eloquent brain that lies beyond that border. Truly then, the first is made more relevant by the second and is contextually dependent on location. Still, the confidence that could come with "foolproof" demarcation of tumor borders intraoperatively (and importantly in real time) offers assuredly intrinsic value to the surgeon, some would likely advance, independent of arguments surrounding statistical connections between EOR and outcomes. Importantly, such demarcation is perhaps increasingly a realizable goal, and, in addition to advances amidst technologies, such as intraoperative MRI and ultrasound, a burgeoning nidus for its realization may lie with fluorescence-guided surgery.

It is near common knowledge now that 5-aminolevulinic acid (5-ALA) is approved for use in Canada, Europe, and Japan, and is undergoing clinical testing presently in the US. What is less recognized, however, are the alternative compounds under examination; their relative mechanisms, advantages, and limitations; their impact for objective outcome measures; and the information needed to properly assess them in isolation or in tandem. Notably, one alternative to 5-ALA is a dye long familiar to the neurosurgical field: fluorescein sodium, whose seemingly specific accumulation within gliomas has been recognized admittedly since the 1940s, and whose testing in humans has reached a new advent in the wake of growing attention for fluorescent guidance.

In this issue of the Journal of Neurosurgery, Diaz et al. advance a degree of new insight into the "old" mechanism for fluorescein's glioma homing. ${ }^{2}$ Indeed, it is similarly long-presumed and noted that fluorescein accumulates in areas of disrupted blood-brain barrier (BBB), but Diaz et al. began with a hypothesis that the dye would perhaps additionally specifically label glioma cells. What they found instead, perhaps not surprisingly, was that the dye accumulated merely in the extracellular spaces around glioma cells in vitro and in vivo, with their further experiments suggesting passive accumulation within areas of BBB disruption.

How should we evaluate the impact and context for this study? On the most apparent level, this study provides evidence for a "passive" means for fluorescein glioma "labeling," whose accuracy is then dependent upon evidence for BBB disruption in glioma and other tumors that are brain situated. This should be contrasted with 5-ALA, which "actively" labels neoplastic cells based upon its metabolic conversion to the fluorescent molecule protoporphyrin IX (PpIX), a heme-synthesis pathway substrate that accumulates preferentially within certain tumor cells, including those of high-grade gliomas.,11 The authors and other advocates of fluorescein, however, will be encouraged by recent work that more definitively characterizes the phenomenon of BBB disruption in glioma and may indirectly support glioma specificity for fluorescein as a result. Watkins et al., in a 2014 study, directly visualized the displacement of astrocytic foot processes from preexisting vascular endothelium by glioma cells, which highly associated with blood vessels, particularly when migrating away from the main mass (i.e., the invasive components). ${ }^{10}$ Furthermore, focal disruptions in the BBB were precipitated by even a single perivascular glioma cell. Although a leaky BBB is historically recognized among newly formed glioma vessels lacking tight junction proteins, this focal disruption of a previously intact BBB was unprecedented, as were the forward disruptions solicited by distant invading cells. If indeed fluorescein proves a sensitive indicator for BBB disruption, these findings imply that it may be a capable 
marker for "forward" areas of microinvasion. Conversely, its freedoms within the extracellular space may instead permit it to diffuse along white matter tracts and stain edematous normal parenchyma, perhaps severely limiting its specificity.

Truthfully, the deeper questions that studies such as Diaz et al. elicit are for fluorescein sodium and for fluorescenceguided surgery more broadly: 1) Can these compounds veritably improve resection? 2) If so, will improving resection translate to improved outcomes? 3) How ultimately should we evaluate and/or compare fluorescence guidance systems? 4) Will implementation be feasible? ${ }^{2}$

Regarding the first question, the only related and reliable data in patients to date come from clinical testing of 5-ALA, which indeed does appear to possess the capacity for improving EOR. A meta-analysis of the literature on 5-ALA, following the guidelines of the Cochrane Collaboration, ultimately selected 5 studies for analysis and concluded significant benefit with regard to EOR, 6-month progression-free survival (PFS), and even overall survival (OS). ${ }^{11}$ The most prominent individual 5-ALA study (also included in the meta-analysis) is of course Stummer's Phase III trial published in 2006, in which 322 patients with newly diagnosed glioma were randomized to either conventional white light microsurgery or 5-ALA-guided resection. ${ }^{8}$ 5-ALA improved the rate of GTR from $36 \%$ in white light controls to 65\%, and the 6-month PFS from $21.1 \%$ to $41.0 \%$. OS did not reach significance $(p=0.1)$, reflecting lack of power to detect an effect. ${ }^{8}$ The study has been criticized for not permitting intraoperative use of neuronavigation (in actuality, neuronavigation was permitted for tumor localization, but not resection; however, many cite this fault as a reason for the low GTR rate in the white light group); for utilizing PFS as a primary end point; and for the likely unavoidable non-blinding of surgeons. An ongoing trial of 5-ALA in the US (BALANCE, clinicaltrials.gov: NCT01502280) permits the use of neuronavigation and may provide further insight into utility in the context of standard/optimized surgical conditions.

No such analysis is available for fluorescein, which remains in feasibility testing, particularly when used in conjunction with a newly designed, and likely mandatory, Yellow 560-nm microscope filter that permits better visualization at lower dosages (clinicaltrials.gov: NCT02028325). ${ }^{7}$ As a molecule with innate fluorescent properties, however, fluorescein can (in contrast to 5-ALA) be visualized at high doses under standard white light without the aid of a filter and has been formally studied in that capacity since the 1990s as a resection aid. A nonrandomized prospective study of use in this context was published in 2003 and demonstrated GTR rates in the fluorescein group of $84.4 \%$, compared to only $30.1 \%$ in controls. No survival benefit was revealed (the study enrolled just 32 patients, however). Similar results were obtained in subsequent studies. ${ }^{3}$ Ultimately, large prospective randomized trials are still wanted for any application of fluorescein guidance.

On the second question of links between EOR and outcomes (the objective utility for fluorescent-guidance is seemingly inevitably tied to this question), a full discussion of this argument is likely beyond the scope of this editorial but is, needless to say, speckled throughout the neu- rosurgical literature and deserves comment. The greatest limitation for resolution is that prospective randomization of glioma patients to subtotal versus gross-total resection is unlikely to ever be accomplished, although in viciously cyclic fashion, ethical arguments preventing such studies are inextricably founded in a "gestalt" that has not been satisfactorily backed by the literature. Through a number of retrospective observational studies, EOR has been identified as a predictor of survival in newly diagnosed ${ }^{6}$ and recurrent ${ }^{1}$ high-grade gliomas. In general, the strongest evidence for this association comes with complete resection, ${ }^{4,8}$ although one well-known study demonstrated a benefit, at least in newly diagnosed glioblastoma, of any resection greater than $78 \% .{ }^{6}$ Others have demonstrated a continuous nonlinear association with EOR and survival, ${ }^{5}$ suggesting that even a less rigid resection threshold might be adoptable for these tumors.

Based on these data, EOR has perhaps mistakenly taken a place alongside age and Karnofsky Performance Scale (KPS) as being a "cause" of survival in this patient population. A "predictor" of survival, however, must be differentiated from a "cause." For example, a younger age, or a better KPS score, may push surgical decision making toward achieving a greater EOR, and thus age or performance status may directly influence (as an upstream determinant) the degree of resection. Such is the limitation of retrospective observational studies. As Barker pointed out in his comments on Sanai and Berger's article, ${ }^{6}$ these studies are often confounded by indication, as the "extent of resection" is frequently determined in turn by the tumor's "resectability," a limitation typically left unaddressed in a nonrandomized study. Of importance, these biases are not fixed by a multivariate analysis, which will still demonstrate EOR to be important, insomuch as the degree of resection was in turn influenced by patient age or performance status. Barker also pointed out that there is a selective loss to follow-up of those patients who do poorly after surgery. ${ }^{6}$ Ironically, some of these biases are well revealed in Stummer's 5-ALA trial, where the age of patients proved lower overall in those receiving complete resection, a subgroup that also proved to have fewer tumors located near eloquent cortex. ${ }^{8}$ This imbalance would typically confound any extrapolation of the data on EOR to a survival end point. What all this means for fluorescent guidance, in the end, is that EOR, much like PFS, may be a somewhat misleading primary end point for study if we are going to require objective outcome-driven justification for its use. Compounding the issue, the most encouraging correlative data between EOR and outcome is likely for low-grade glioma, where neither 5-ALA nor fluorescein proves useful.

The next question then does indeed become how to properly evaluate and compare such technology. The lowhanging fruit is safety, concerns for which to date have mostly surrounded the consequences of obtaining more complete and aggressive resections, including into areas of lower fluorescence whose specificity for tumor remains somewhat indeterminate, as well as dependent on the agent used. If we search instead for acceptable objective outcomes measures, we may be left chasing the grail of overall survival differences, which similarly becomes difficult to adequately design studies to reveal. If easier-to-obtain 
surrogates such as PFS and EOR leave us with a bad taste, is it wrong to assume an intrinsic value to a technology that provides immeasurables, such as surgeon confidence? Can safety, sensitivity, and specificity suffice when comparing fluorescent contrast agents, an increasing number of which are in preclinical testing (i.e., immunoliposomes, indocyanine green, Cy5.5-chlorotoxin, and quantum dots)? There are exceedingly few studies backing the overall survival benefit to patients for the use of universal technologies such as the operating microscope, ultrasound, or neuronavigation, for instance. The difference here of course is that fluorescence-guided surgery necessitates the administration of a drug, which, in turn, eventually requires FDA approval. To that argument, however, these are merely contrast agents, and a literature search similarly yields no studies revealing the overall survival benefit to gadolinium or iodinated contrast administration for MRI or CT prior to glioma surgery (and notably, both have well-documented toxicities). In the end, the final question of feasibility within the US will be linked to FDA requirements for approval, but as a field, we likely only need find an acceptable method for contrasting contrasts.

http://thejns.org/doi/abs/10.3171/2014.10.JNS141788

\section{References}

1. Bloch O, Han SJ, Cha S, Sun MZ, Aghi MK, McDermott MW, et al: Impact of extent of resection for recurrent glioblastoma on overall survival. Clinical article. J Neurosurg 117:1032-1038, 2012

2. Diaz RJ, Dios RR, Hattab EM, Burrell K, Rakopoulos P, Sabha N, et al: Study of the biodistribution of fluorescein in glioma-infiltrated mouse brain and histopathological correlation of intraoperative findings in high-grade gliomas resected under fluorescein fluorescence guidance. J Neurosurg [epub ahead of print April 3, 2015. DOI: 10.3171/2015.2.JNS132507]

3. Koc K, Anik I, Cabuk B, Ceylan S: Fluorescein sodiumguided surgery in glioblastoma multiforme: a prospective evaluation. Br J Neurosurg 22:99-103, 2008

4. Lacroix M, Abi-Said D, Fourney DR, Gokaslan ZL, Shi W, DeMonte F, et al: A multivariate analysis of 416 patients with glioblastoma multiforme: prognosis, extent of resection, and survival. J Neurosurg 95:190-198, 2001

5. Marko NF, Weil RJ, Schroeder JL, Lang FF, Suki D, Sawaya RE: Extent of resection of glioblastoma revisited: personalized survival modeling facilitates more accurate survival prediction and supports a maximum-safe-resection approach to surgery. J Clin Oncol 32:774-782, 2014

6. Sanai N, Berger MS: Glioma extent of resection and its impact on patient outcome. Neurosurgery 62:753-764, 2008

7. Schebesch KM, Proescholdt M, Höhne J, Hohenberger C, Hansen E, Riemenschneider MJ, et al: Sodium fluoresceinguided resection under the YELLOW $560 \mathrm{~nm}$ surgical microscope filter in malignant brain tumor surgery-a feasibility study. Acta Neurochir (Wien) 155:693-699, 2013

8. Stummer W, Pichlmeier U, Meinel T, Wiestler OD, Zanella F, Reulen HJ, et al: Fluorescence-guided surgery with 5-aminolevulinic acid for resection of malignant glioma: a randomised controlled multicentre phase III trial. Lancet Oncol 7:392-401, 2006

9. Stummer W, Stepp H, Möller G, Ehrhardt A, Leonhard M, Reulen HJ: Technical principles for protoporphyrin-IX-fluorescence guided microsurgical resection of malignant glioma tissue. Acta Neurochir (Wien) 140:995-1000, 1998

10. Watkins S, Robel S, Kimbrough IF, Robert SM, Ellis-Davies $\mathrm{G}$, Sontheimer H: Disruption of astrocyte-vascular coupling and the blood-brain barrier by invading glioma cells. Nat Commun 5:4196, 2014

11. Zhao S, Wu J, Wang C, Liu H, Dong X, Shi C, et al: Intraoperative fluorescence-guided resection of high-grade malignant gliomas using 5-aminolevulinic acid-induced porphyrins: a systematic review and meta-analysis of prospective studies. PLoS One 8:e63682, 2013

\section{Response}

\section{Roberto Jose Diaz, MD, PhD, '1 Gelareh Zadeh MD, PhD, 3,4 James T. Rutka, MD, PhD, ${ }^{2-4}$ and Aaron A. Cohen-Gadol, MD, MSc ${ }^{5,6}$}

\begin{abstract}
1Department of Clinical Neurosciences, University of Calgary, Alberta; ${ }^{2}$ Arthur and Sonia Labatt Brain Tumour Research Centre, The Hospital for Sick Children; ${ }^{3}$ Department of Laboratory Medicine \& Pathobiology and ${ }^{4}$ Division of Neurosurgery, Department of Surgery, University of

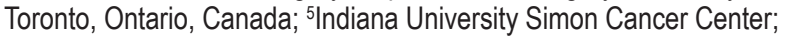
and ${ }^{6}$ Goodman Campbell Brain and Spine, Department of Neurological Surgery, Indiana University, Indianapolis, Indiana
\end{abstract}

We sincerely appreciate the thoughtful comments of Dr. Sampson and his team. Their expert comments have advanced the impact of our work. In fact, we believe their editorial is a reference in its own right and a candid evaluation of current technologies and future directions for intraoperative tumor fluorescence.

Intraoperative neuronavigation is presently a staple of brain tumor surgery because of its ability to enhance the surgeon's confidence and reduce surgical planning errors. Although neuronavigation is a helpful tool, it has limitations for accurate intraoperative tumor localization. Resection of parenchymal tumors may involve significant brain shift due to progressive tumor removal, CSF volume fluctuations, and release of associated cyst contents. During the final stages and more critical parts of the operation, the surgeon may rely on prior experience involving tissue texture, consistency, and color, along with anatomical landmarks, to complete the resection safely and effectively. Other strategies used to address the limitations of neuronavigation include intraoperative $\mathrm{MRI}^{6}$ and ultrasonography. ${ }^{4}$ With the advent of fluorescence-guided microscopic surgery, we are at an important evolutionary point in overcoming the limitations of neuronavigation.

Fluorescence-guided surgery combines real-time microscopic visualization of fluorophores with passive or active tumor labeling properties to allow greater confidence in defining tumor margins despite the challenges of intraoperative brain shift. Proof of principle in maximizing the EOR has been provided by Stummer et al. ${ }^{7}$ for 5-ALA labeling of glioblastoma. In our article, we now report on the role of fluorescein in delineating the regions of $\mathrm{BBB}$ breakdown in glioblastoma surgery and its potential for maximizing the EOR.

Undoubtedly, further work to demonstrate the effectiveness of fluorescein-guided glioblastoma resection is required and planned. This will invariably involve the coordinated effort of multiple centers to accrue significant numbers of patients in a randomized fashion, comparing resection using standard neuronavigation versus standard neuronavigation plus fluorescein wide-field microscopy. 
Careful selection of the primary outcome variables in such a study will have great value.

Should the EOR determine by itself the utility of this agent? Or should we ask for more, such as improvements in PFS or OR? The current literature suggests that improvements in the EOR would be easily attainable with fluorescence-guided glioblastoma resection. And the results of a controlled randomized study in which patient age and KPS are balanced between groups would provide more information about the influence of resection on PFS and OS for glioblastoma. As mentioned by Dr. Sampson, the realization of this trial is potentially too ambitious. Of interest would be the impact of the EOR and changes in PFS on the quality of life for these patients. Recently, Jakola et al. ${ }^{2}$ reported an association between the use of intraoperative ultrasound and preservation of quality of life (as measured by the EQ-5D quality of life index) in glioma patients. It remains to be seen if similar effects will be observed with fluorescence-guided surgery.

We are cognizant that 5-ALA and fluorescein are only the tip of the iceberg of clinically relevant biological imaging agents. Although 5-ALA is more specific or active for tumor labeling, both 5-ALA and fluorescein use the same mechanism, the compromised $\mathrm{BBB}$, to reach the tumor. The regulatory barriers and the unreliable availability of 5-ALA have significantly limited its use in this country. Fluorescein fluorescence is a very viable and readily accessible option. Importantly, the subjective fluorescence at the tumor margin for both contrast agents, partially accounted for by optical properties of fluorescence, is another nuance when using this technology. More complex agents include fluorescent and surface-enhanced Raman scattering nanoparticles with the potential for cell-specific tumor identification. ${ }^{3,5}$ The technique of labeling white matter tracts with myelin-specific fluorophores, currently under development, ${ }^{1}$ is expected to further expand the application of fluorescence-guided surgery with definition of anatomical boundaries for safe resection. Certainly the safety and usefulness of molecular imaging agents will be a focus of further research in the laboratory and the clinical setting.

The evolving field of fluorescence-guided brain tumor surgery is a stepping stone to new and exciting possibilities for brain tumor diagnosis and therapy. Given the molecular and clinical diversity of glioblastoma, it is possible that the EOR will not be the primary determinant of outcome. Therefore, the development of technology that can lead to new therapeutic strategies is warranted. These factors are reflected in the trials noted by Dr. Sampson's editorial, despite the shortcoming of these trials.

Maximal safe resection is currently our goal, but it remains to be seen whether this philosophical tenet will remain in the future as new molecular therapies are developed. It is important to emphasize that major breakthroughs in glioma care are not based on fluorescent technologies, but will originate from innovative and progressive laboratories such as those led by Dr. Sampson.

\section{References}

1. Gibbs-Strauss SL, Nasr KA, Fish KM, Khullar O, Ashitate Y, Siclovan TM, et al: Nerve-highlighting fluorescent contrast agents for image-guided surgery. Mol Imaging 10:91-101, 2011

2. Jakola AS, Unsgård G, Solheim O: Quality of life in patients with intracranial gliomas: the impact of modern image-guided surgery. J Neurosurg 114:1622-1630, 2011

3. Kircher MF, de la Zerda A, Jokerst JV, Zavaleta CL, Kempen PJ, Mittra E, et al: A brain tumor molecular imaging strategy using a new triple-modality MRI-photoacoustic-Raman nanoparticle. Nat Med 18:829-834, 2012

4. Prada F, Del Bene M, Mattei L, Casali C, Filippini A, Legnani F, et al: Fusion imaging for intra-operative ultrasoundbased navigation in neurosurgery. J Ultrasound 17:243-251, 2014

5. Qian X, Peng XH, Ansari DO, Yin-Goen Q, Chen GZ, Shin $\mathrm{DM}$, et al: In vivo tumor targeting and spectroscopic detection with surface-enhanced Raman nanoparticle tags. Nat Biotechnol 26:83-90, 2008

6. Senft C, Bink A, Franz K, Vatter H, Gasser T, Seifert V: Intraoperative MRI guidance and extent of resection in glioma surgery: a randomised, controlled trial. Lancet Oncol 12:997-1003, 2011

7. Stummer W, Pichlmeier U, Meinel T, Wiestler OD, Zanella F, Reulen HJ, et al: Fluorescence-guided surgery with 5-aminolevulinic acid for resection of malignant glioma: a randomised controlled multicentre phase III trial. Lancet Oncol 7:392-401, 2006 